\title{
Clinical and morphological features of patients who underwent endovascular interventions for lower extremity arterial occlusive diseases
}

\author{
Sakir Arslan, Isa Oner Yuksel, Erkan Koklu, Goksel Cagirci, Cagin Mustafa Ureyen, Nermin Bayar, \\ Selcuk Kucukseymen, Gorkem Kus
}

Antalya Training and Research Hospital, Antalya, Turkey

Postep Kardiol Inter 2015; 11, 2 (40): 114-118

DOI: $10.5114 /$ pwki.2015.52284

\begin{abstract}
A bstract
Introduction: Patients with peripheral arterial disease (PAD) are at increased risk for all-cause mortality and cardiovascular mortality.

Aim: To present anatomical and morphological characteristics of patients who underwent endovascular stenting with laboratory and our mid-term results.

Material and methods: One hundred fifty-three patients (mean age: $62.8,86 \%$ male) who underwent percutaneous intervention of lower extremity arteries were included in the study. Demographic characteristics, medical history, physical examination and laboratory findings of patients were analyzed. Patients' lesions were classified according to the TransAtlantic Inter-Society Consensus (TASC). Clinical outcomes included complications and mortality, 6-minute walking distance, functional class (NYHA) and patency rates.

Results: Seventy percent of patients had hypertension, $42 \%$ were smokers, $78 \%$ had coronary artery disease, $20 \%$ had coronary artery bypass grafting, $55 \%$ had diabetes mellitus and $71 \%$ had dyslipidemia. Six patients with diabetes mellitus and poor wound healing despite medical therapy were treated with stenting leading to alleviation of pain and avoidance of amputation. The initial technical success rate of revascularization was 95.6\% (153/160). Our mid-term results show that percutaneous procedures in lower extremity arterial diseases can be performed with low complication and high success rates. Patients' 6-minute walk distance, ankle/ brachial index values, functional class and the status of foot ulcers were evaluated.

Conclusions: Especially in patients with distal vascular disease, poor wound healing and no chance of surgical revascularization, percutaneous endovascular revascularization may provide good blood flow and prevent amputation.
\end{abstract}

Key words: peripheral arterial disease, endovascular techniques, stents.

\section{Introduction}

The use of endovascular interventions for lower extremity ischemia has increased in the past decade. Peripheral arterial occlusive disease (PAOD) is frequent and underdiagnosed [1]. If PAOD is not diagnosed in time and treatment is not initiated immediately, the probability of disease progression and development of critical limb ischemia is higher [2]. Although treatment options, including medical, surgical and endovascular intervention possibilities, have been improved in the last decades, amputation and mortality rates are still high $[3,4]$.

In recent years, an increasing number of patients have undergone percutaneous intervention as a first line therapy for arterial occlusive disease of the lower extremity [5].
Painful vascular ulcers and intermittent claudication greatly reduce mobility and quality of life. Successful revascularization could reduce the rate of amputation, improve the healing of foot ulcers and raise the quality of life in PAOD.

\section{Aim}

In the study, we aimed to evaluate lower extremity endovascular revascularization therapy efficacy, feasibility, complications, anatomical and demographical characterization of PAOD patients.

\section{Material and methods}

We included 423 consecutive PAOD patients treated at our hospital from 2011 to 2014 in our retrospective

\section{Corresponding author:}

Selcuk Kucukseymen MD, Antalya Training and Research Hospital, Varlık Mahallesi Kazım Karabekir Caddesi Soğuksu 07100 Antalya, Turkey, fax: +90 5074315691, e-mail: skucukseymen@gmail.com

Received: 3.10.2014, accepted: 9.01.2015. 
data analysis. Endovascular revascularization was performed in 153 patients between May 2011 and April 2014 in our center because of intermittent claudication or critical limb ischemia. Patients with demographic features, comorbidities, indications for intervention, and laboratory data were recorded. Concomitant coronary angiography was performed in patients with PAOD undergoing peripheral endovascular intervention.

Significant coronary artery disease (CAD) was defined as the presence of at least one lesion with $\geq 50 \%$ luminal stenosis by quantitative coronary angiography. Lesions with $<50 \%$ stenosis are not defined as significant CAD; they were considered minimal CAD. Chronic kidney disease was defined as the presence, for at least three months, of an estimated glomerular filtration rate (eGFR) less than $60 \mathrm{ml} / \mathrm{min} / 1.73 \mathrm{~m}^{2}$.

The diagnosis of PAOD was assigned in our outpatient clinic by means of clinical evaluation, ankle brachial index $(A B I)$, and duplex ultrasonography. The $A B I$ is calculated by dividing the ankle systolic blood pressure by the arm systolic blood pressure. When patients were admitted to the hospital, medical records of the patients were analyzed with attention to cardiovascular risk factors and comorbidities. Blood was taken in fasting patients and laboratory examinations were performed.

Indications for an interventional procedure included intermittent claudication or limb-threatening ischemia detected by peripheral angiography. Interventions were performed percutaneously from a femoral or brachial approach using 6-Fr or 8-Fr sheaths. Digital subtraction angiography was subsequently performed to evaluate lesion morphology and the status of inflow and outflow. Predilatation with undersize balloons was performed restrictively in patients with a very tight occlusion or heavily calcified lesion that did not allow primary passage of the stent introducer device. A contralateral femoral approach and left brachial approach was used in the majority of patients. In rare cases, in below the knee interventions, an ipsilateral antegrade femoral approach was used. Percutaneous interventions were performed through an $8 \mathrm{Fr}$ sheath for a femoral approach and a $6 \mathrm{Fr}$ sheath for a brachial approach. By the femoral approach, the guiding sheath (length 55-90 cm) was advanced through an $8 \mathrm{Fr}$ sheath. However, it was advanced without a sheath in the brachial approach. All patients were anticoagulated with intravenous unfractionated heparin and followed by activated clotting time (ACT). Monitoring with fluoroscopy, stenotic segments were crossed with a hydrophilic guidewire (0.035 inch) while a total occlusion was crossed (support with $4 \mathrm{Fr}$ microcatheter) with either luminal or subintimal technique using guide wires (0.018 inch Treasure floppy or edge weight $12 \mathrm{~g}$ guide wires). After confirmation of the wire in the distal lumen angiographically, percutaneous transluminal angioplasty (PTA) was performed with balloons (2.5-7.0 $\mathrm{mm}$ diameter) for the target vessel. Inflation times ranged from 60 to $120 \mathrm{~s}$ at
6 to $14 \mathrm{~atm}$ of pressure. We determined the stent diameter and inflation atmosphere according to the distal diameter of the target vessel. Then the stent was implanted. After the intervention, distal run-off and presence of any residual stenosis were checked with the images obtained from control angiography. The PTA and stent technique success was defined as a residual stenosis of $\leq 30 \%$. All percutaneous interventions were performed under local anesthesia. A total of 215 stents were implanted into 163 limbs (average: 1.31 stents/limb). Acetyl salicylic acid $100 \mathrm{mg}$ was administered daily to all patients after endovascular recanalization unless contraindicated. A loading dose of clopidogrel $300 \mathrm{mg}$ was administered in the ward, followed by $75 \mathrm{mg}$ per day for 1 year.

Assessments were performed at baseline and the time of discharge, and follow-up lasted for 1-31 months (mean $12.72 \pm 7.9$ months). Patients after 6 months of intervention were evaluated by physical examination (pulses and presence or absence of claudication), ABI and 6-minute walk test. When indicated, angiography was performed for angiographic assessment of restenosis. The study was approved by the local review board and ethics committee.

\section{Statistical analysis}

Clinical characteristics of patients were analyzed using descriptive statistics. The categorical variables were described with percentages and frequencies. Continuous variables were described with means and standard deviations. A $p$ value of $<0.05$ was accepted as statistically significant. For the statistical analysis the SPSS 16.0 (Statistical Package for Social Science-SPSS, Inc., Chicago, Illinois, USA) program was used.

\section{Results}

A total of 153 PAOD patients were included in the current analysis. During the 3 years of study, a total of 163 limbs from 153 patients (132 men, ranging from 36 to 85 years of age; mean \pm standard deviation, $62.88 \pm 9.46$ years) received nitinol stent-assisted angioplasty in our center. Baseline characteristics of the patients are summarized in Table I.

In 31 patients, there was total occlusion, and the process was performed using a microcatheter with a 0.018inch guide wire and a mean lesion length of $74.76 \pm 48 \mathrm{~mm}$. Calcification was observed in $34.6 \%$ of the lesions (53/153). All lesions were categorized by location and TransAtlantic Inter-Society Consensus (TASC): 99 (64.7\%) were iliac lesions, 45 (29.4\%) were femoral lesions, 9 (5.9\%) were below the knee lesions. Thirty-one (20\%) patients had total occlusion. Details of the lesions are shown in Table II. In 85 (55.6\%) patients balloon expandable stents, and for 54 (35.3\%) patients self-expandable stents were used. In 12 patients, balloon angioplasty and/or stenting was performed for popliteal-below the 
Table I. Characteristics of all PAOD patients included in the study

\begin{tabular}{lc} 
Parameter & Results \\
\hline Age, mean [years] & $62.8(36-85)$ \\
\hline Men, $n(\%)$ & $132(86)$ \\
\hline Hypertension, $n(\%)$ & $107(70)$ \\
\hline Diabetes mellitus, $n(\%)$ & $84(55)$ \\
\hline Smoking, $n$ (\%) & $64(42)$ \\
\hline Chronic kidney disease, $n$ (\%) & $52(34.3)$ \\
\hline Congestive heart failure, $n(\%)$ & $20(13)$ \\
\hline Significant coronary artery disease, $n$ (\%) & $119(78)$ \\
\hline Prior CABG, $n$ (\%) & $30(20)$ \\
\hline Previous stroke, $n$ (\%) & $17(11)$ \\
\hline Intermittent claudication, chief complaint, $n(\%)$ & $128(83.7)$ \\
\hline Foot wounds, chief complaint, $n$ (\%) & $25(16.3)$
\end{tabular}

Table II. Lesion properties

\begin{tabular}{lc} 
Property & Value \\
\hline Self expandable stent lesion length $[\mathrm{cm}]$ & $\begin{array}{c}9.78 \pm 5.5 \\
\text { (range: } 2.0-22.9)\end{array}$ \\
\hline Balloon expandable stent lesion length $[\mathrm{cm}]$ & $\begin{array}{c}5.64 \pm 3.1 \\
\text { (range: } 1.8-17.3)\end{array}$ \\
\hline TASC A, $n(\%)$ & $42(27.5)$ \\
\hline TASC B, $n(\%)$ & $72(47.0)$ \\
\hline TASC C, $n(\%)$ & $38(24.8)$ \\
\hline TASC D, $n(\%)$ & $1(0.7)$ \\
\hline Total occlusion, $n(\%)$ & $31(20)$ \\
\hline Calcification, $n(\%)$ & $53(34.6)$
\end{tabular}

knee vessels. In 2 patients, drug-eluting stents used in coronary intervention were implanted in the posterior tibial artery and anterior tibial artery.

The interventional procedure success rate was $95.6 \%$ (153/160). No perioperative death occurred. Technical and clinical success, which was defined as a residual stenosis of $\leq 30 \%$ by visual estimate, was obtained in 153 of 160 procedures.

In 7 patients, the procedure was not performed due to technical challenges. One patient could not undergo the procedure due to dissection in the antegrade approach. In 3 patients we failed to cross the total occlusion with a wire. Therefore, these patients were operated on for revascularization. The overall in-hospital complication rate was $7.8 \%$ in the endovascular treatment. Procedure-related complications occurred in 12 procedures, including 6 groin and brachial hematomas, 2 pseudoan-
Table III. Details and complications of 165 interventions

\begin{tabular}{|c|c|}
\hline Interventional details & Value, $n(\%)$ \\
\hline Angioplasty alone (without stent) & $5(3.3)$ \\
\hline \multicolumn{2}{|l|}{ Number of stents: } \\
\hline 1 & $94(61.4)$ \\
\hline 2 & $43(28.1)$ \\
\hline 3 & $9(5.9)$ \\
\hline 4 & $2(1.3)$ \\
\hline Iliac stenosis & $99(64.7)$ \\
\hline SFA stenosis & $45(29.4)$ \\
\hline Infrapopliteal stenosis & $9(5.9)$ \\
\hline Predilatation & $44(29.1)$ \\
\hline Postdilatation & $64(42.1)$ \\
\hline Technical success & $153 / 160(95.6)$ \\
\hline Balloon expandable stent & $85(57.4)$ \\
\hline Self expandable stent & $54(36.5)$ \\
\hline Combined (balloon and self expandable) & $7(4.7)$ \\
\hline Drug-eluting stent (coronary) & $2(1.4)$ \\
\hline \multicolumn{2}{|l|}{ Complications: } \\
\hline Distal embolism & 0 \\
\hline Vessel rupture & $1(0.6)$ \\
\hline Vessel dissection & $2(1.3)$ \\
\hline Hematoma & $6(3.9)$ \\
\hline Pseudoaneurysm & $2(1.3)$ \\
\hline Fistula (aortocaval) & $1(0.6)$ \\
\hline Total & $12(7.84)$ \\
\hline
\end{tabular}

eurysms, and 2 vessel dissections. All complications were successfully treated at the time of the primary procedure. Details of the interventions and complications are shown in Table III.

Intraoperative and perioperative 30-day mortality was $0 \%$. Major complications were access-related and included groin and brachial hematoma in $3.9 \%$ of patients (not requiring operation) and pseudoaneurysm formation in $2(1.3 \%)$ patients (1 patient requiring an operation).

Mean follow-up time was $14.32 \pm 8.7$ months (1-35 months). During follow-up, 5 patients died because of myocardial infarction and 17 patients were lost to follow-up. During follow-up, 3 in-stent restenoses and successful re-interventions with a drug-eluting balloon were performed. In the follow-up, 1 patient who did not benefit from endovascular treatment underwent surgery for amputation of the limb. 
Before endovascular stenting, the 6-minute walk distance of patients averaged $84.71 \pm 62 \mathrm{~m}$; this value after successful intervention rose to $348 \pm 60 \mathrm{~m}$. Before the intervention, while the average $\mathrm{ABI}$ value was $0.49 \pm 0.14$ for the right lower limb, this value was $1.0 \pm 0.13$ after intervention; the increase was statistically significant $(p=0.0001)$. As for the left lower extremity, these values are $0.50 \pm 0.16$ before the procedure and after the procedure $1.00 \pm 0.17(p=0.0001)$. In controls, $A B I$ values of the patients increased, and there was a significant improvement in functional capacity. Six patients were saved from major amputation due to a non-healing foot wound.

\section{Discussion}

Patients with PAOD have a poor clinical outcome. During the first year of diagnosis, mortality is $20-30 \%$, and an equivalent number undergo amputations due to persistent pain [6]. The primary non-invasive test for the diagnosis of lower extremity artery disease (LEAD) is the $A B I$. In healthy persons, $A B I$ is greater than 1.0. Usually $A B I<0.9$ is used to describe LEAD. The sensitivity and specificity have been estimated at $79 \%$ and $96 \%$, respectively [7]. Moreover, the level of ABI correlates with LEAD severity. $A$ reduced $A B I$ has been associated with an increased risk of cardiovascular morbidity and mortality in several studies [8].

In patients with PAOD, the first line therapy has shifted over the past decade from bypass surgery to endovascular intervention. However, bypass surgery can only be performed on a minority of patients without poor runoff, advanced age, and cardiac co-morbidities. The mortality and major morbidity in patients treated for limb-threatening disease with percutaneous intervention was only 0 and $8.1 \%$ respectively. In our study, procedure-related mortality was not observed. There was a minor complication rate of 7.84\%; processing was carried out successfully.

The role of femoral arterial stents in the management of PAOD is still controversial. But compared with percutaneous transluminal angioplasty (PTA), stenting results and patency rates are better. Our study confirmed that endovascular intervention is an effective and safe therapy for lower extremity arterial occlusive disease. Restenotic lesions of the femoropopliteal arterial segment occur more than in the other arterial segments, because these lesions are longer, are exposed to stretching, bending and twisting, and are dense. Predilatation may dramatically reduce the residual stenosis when compared with postdilatation, but it may cause more distal embolization [9]. However, in our study, predilatation was performed only in patients with very tight occlusions or heavily calcified lesions that did not allow primary passage of the stents.

The TASC (TransAtlantic Inter-Society Consensus) recommendation states that angioplasty is the first choice therapy for aortoiliac and femoropopliteal lesions of classes A-C, but not for those of class D [10]. However, in the present studies, when surgery is not an option for patients with TASC D lesions (because of comorbidities), the short and midterm patencies seem to be acceptable.

The treatment of infrapopliteal arterial occlusive disease is still a clinical problem for vascular surgeons and interventional cardiologists. However, in patients with poor wound healing and no chance of surgical revascularization, percutaneous treatment can help by accelerating wound healing and save limbs from amputation. Therefore, when revascularization in the infrapopliteal segments is indicated, an endovascular intervention as a first strategy should be considered.

This was a single center study. The sample of this study was relatively small and the follow-up period was relatively short. In patients who underwent interventions, long-term patency rates are uncertain. There was no control group of patients who received medical therapy and conventional bypass surgery enrolled in this study.

\section{Conclusions}

Percutaneous revascularization has rapidly emerged as an alternative to open bypass surgery for patients with chronic lower extremity arterial disease. In this study, we tried to demonstrate the good mid-term patency rate of percutaneous intervention therapy and low risk of morbidity and mortality.

\section{Conflict of interest}

The authors declare no conflict of interest.

\section{References}

1. Bertomeu V, Morillas P, Gonzalez-Jaunatey JR, et al. Prevalence and prognostic influence of peripheral arterial disease in patients $>$ or $=40$ years old admitted into hospital following and acute coronary event. Eur J Vasc Endovasc Surg 2008; 36: 189-96.

2. Norgren L, Hiatt WR, Dormandy JA, et al. Inter-Society Consensus for the Management of Peripheral Arterial Disease (TASC II). J Vasc Surg 2007; 45 Suppl S: S5-67.

3. Novo S, Coppola G, Milio G. Critical limb ischemia: definition and natural history. Curr Drug Targets Cardiovasc Haematol Disord 2004; 4: 219-25.

4. Bertele V, Roncaglioni MC, Pangrazzi J, et al. Clinical outcome and its predictors in 1560 patients with critical leg ischemia. Chronic Critical Leg Ischemia Group. Eur J Vasc Endovasc Surg 1999; 18: 401-10.

5. Derubertis BG, Pierce M, Ryer EJ, et al. Reduced primary patency rate in diabetic patients after percutaneous intervention results from more frequent presentation with limb-threatening ischemia. J Vasc Surg 2008; 47: 101-8.

6. Dormandy JA, Rutherford RB. Management of peripheral arterial disease (PAD). TASC working group. J Vasc Surg 2000; 31: 1-296.

7. Lijmer JG, Hunink MG, van den Dungen JJ, et al. ROC analysis of noninvasive tests for peripheral arterial diseases. Ultrasound Med Biol 1996; 22: 391-8.

8. Fowkes FG, Price JF, Stewart MC, et al. Aspirin for prevention of cardiovascular events in a general population screened for a low 
ankle brachial index: a randomized controlled trial. JAMA 2010; 303: 841-8.

9. Lenti M, Cieri E, De Rango P, et al. Endovascular treatment of long lesions of the superficial femoral artery: result from a multicenter registry of a spiral, covered polytetrafluoroethylene stent. J Vasc Surg 2007; 45: 32-9.

10. European Stroke Organisation, Tendera M, Aboyans V, et al. The Tasc Force on the Diagnosis and Treatment of Peripheral Artery Diseases of the European Society of Cardiology (ESC). Eur Heart J 2011; 32: 2851-906. 